\title{
A Comparative Study of Variational Iteration Method and He-Laplace Method
}

\author{
Hradyesh Kumar Mishra \\ Department of Mathematics, Jaypee University of Engineering \& Technology, Guna, India \\ Email: hk.mishra@juet.ac.in
}

Received August 6, 2012; revised September 6, 2012; accepted September 13, 2012

\begin{abstract}
In this paper, variational iteration method and He-Laplace method are used to solve the nonlinear ordinary and partial differential equations. Laplace transformation with the homotopy perturbation method is called He-Laplace method. A comparison is made among variational iteration method and He-Laplace. It is shown that, in He-Laplace method, the nonlinear terms of differential equation can be easily handled by the use of He's polynomials and provides better results.
\end{abstract}

Keywords: Variational Iteration Method; He-Laplace Transform Method; Homotopy Perturbation Method; Ordinary Differential Equation; Partial Differential Equations; He's Polynomials

\section{Introduction}

Nonlinearity exists everywhere and nature is nonlinear in general. The search for a better and easy to use tool for the solution of nonlinear equations that illuminate the nonlinear phenomena of real life problems of science and engineering has recently received a continuing interest. Various methods, therefore, were proposed to find approximate solutions of nonlinear equations. Some of the classical analytic methods are Lyapunov's artificial small parameter method [1], perturbation techniques $[2,3]$, $\delta$-expansion method [4] and Hirota bilinear method [5-6]. In recent years, many authors have paid attention to study the solutions of nonlinear partial diffenential equations by using various methods. Among these are the Adomian decomposition method (ADM) [7], He's Semiinverse method [8], the tanh method, homotopy perturbation method(HPM), Sinh-Cosh method, the differential transform method and the variational iteration method (VIM) [9-17]. Several techniques including the Adomian decomposition method, the variational iteration method, the weighted finite difference techniques and the Laplace decomposition method have been used to solve nonlinear differential equations [18-26]. J. H. He developed the homotopy perturbation method (HPM) [27-42] by merging the standard homotopy and perturbation for solving various physical problems. The Laplace transform is totally incapable of handling nonlinear equations because of the difficulties that are caused by the nonlinear terms. Various ways have been proposed recently to deal with these nonlinearities such as the Adomian decomposition method [43] and the Laplace decomposition algorithm [44-48]. Furthermore, the homotopy perturbation method is also combined with the well-known Laplace transformation method [49] which is known as He-Laplace method.

In this paper, the main objective is to introduce a comparative study to nonlinear ordinary differential equation and partial differential equations by using variational iteration method and He-Laplace method.

It is worth mentioning that He-Laplace method is an elegant combination of the Laplace transformation, the homotopy perturbation method and He's polynomials. The use of He's polynomials in the nonlinear term was first introduced by Ghorbani [50]. The proposed algorithm provides the solution in a rapid convergent series which may lead to the solution in a closed form. This paper contains basic idea of homotopy perturbation method in Section 2, variational iteration method in Section 3, Laplace homotopy perturbation method in Section 4 and conclusions in Section 5 respectively.

\section{Basic Idea of Homotopy Perturbation Method and He-Laplace Method}

\subsection{Homotopy Perturbation Method}

Consider the following nonlinear differential equation

$$
A(y)-f(r)=0, \quad r \in \Omega
$$

with the boundary conditions of

$$
B\left(y, \frac{\partial y}{\partial n}\right)=0, \quad r \in \Gamma
$$


where A, B, $f(r)$ and $\Gamma$ are a general differential operator, a boundary operator, a known analytic function and the boundary of the domain $\Omega$, respectively.

The operator A can generally be divided into a linear part $\mathrm{L}$ and a nonlinear part N. Equation (1) may therefore be written as:

$$
L(y)+N(y)-f(r)=0
$$

By the homotopy technique, we construct a homotopy $v(r, p): \Omega \times[0,1] \rightarrow R$ which satisfies:

$$
\begin{aligned}
& H(v, p)=(1-p)\left[L(v)-L\left(y_{0}\right)\right] \\
& +p[A(v)-f(r)]=0
\end{aligned}
$$

or

$$
\begin{aligned}
& H(v, p)=L(v)-L\left(y_{0}\right) \\
& +p L\left(y_{0}\right)+p[N(v)-f(r)]=0
\end{aligned}
$$

where $p \in[0,1]$ is an embedding parameter, while $y_{0}$ is an initial approximation of Equation (1), which satisfies the boundary conditions. Obviously, from Equatons (4) and (5), we will have:

$$
\begin{gathered}
H(v, 0)=L(v)-L\left(y_{0}\right)=0 \\
H(v, 1)=A(v)-f(r)=0
\end{gathered}
$$

The changing process of $p$ from zero to unity is just that of $v(r, p)$ from $y_{0}$ to $y(r)$. In topology, this is called deformation, while $L(v)-L\left(y_{0}\right)$ and $A(v)-f(r)$ are called homotopy. If the embedding parameter $p$ is considered as a small parameter, applying the classical perturbation technique, we can assume that the solution of Equations (4) and (5) can be written as a power series in $p$ :

$$
v=v_{0}+p v_{1}+p^{2} v_{2}+p^{3} v_{3}+\cdots \infty
$$

Setting $p=1$ in Equation (8), we have

$$
y=\lim _{p \rightarrow 1} v=v_{0}+v_{1}+v_{2}+\cdots
$$

The combination of the perturbation method and the homotopy method is called the HPM, which eliminates the drawbacks of the traditional perturbation methods while keeping all its advantages. The series (9) is convergent for most cases. However, the convergent rate depends on the nonlinear operator $A(v)$. Moreover, $\mathrm{He}$ [51] made the following suggestions:

1) The second derivative of $N(v)$ with respect to $v$ must be small because the parameter may be relatively large, i.e. $\quad p \rightarrow 1$.

2) The norm of $L^{-1}\left(\frac{\partial N}{\partial v}\right)$ must be smaller than one so that the series converges.

\subsection{He-Laplace Method}

Consider the following nonlinear differential equation (IVP):

$$
\begin{aligned}
& y^{\prime \prime}+p_{1} y^{\prime}+p_{2} y+p_{3} f(y)=f(x) \\
& y(0)=\alpha, \quad y^{\prime}(0)=\beta
\end{aligned}
$$

where $p_{1}, p_{2}, p_{3}, \alpha, \beta$ are constants. $f(y)$ is a nonlinear function and $f(x)$ is the source term. Taking Laplace transformation (denoted throughout this paper by $\mathrm{L}$ ) on both side of Equation (10), we have

$$
\begin{aligned}
& L\left[y^{\prime \prime}\right]+L\left[p_{1} y^{\prime}\right]+L\left[p_{2} y\right]+L\left[p_{3} f(y)\right] \\
& =L[f(x)]
\end{aligned}
$$

By using linearity of Laplace transformation, the result is

$$
\begin{aligned}
& L\left[y^{\prime \prime}\right]+p_{1} L\left[y^{\prime}\right]+p_{2} L[y]+p_{3} L[f(y)] \\
& =L[f(x)]
\end{aligned}
$$

Applying the formula on Laplace transform, we obtain

$$
\begin{aligned}
& s^{2} L[y]-s y(0)-y^{\prime}(0)+p_{1}\{s L[y]-y(0)\} \\
& +p_{2} L[y]+p_{3} L[f(y)]=L[f(x)]
\end{aligned}
$$

Using initial conditions in Equation (14), we have

$$
\begin{aligned}
& \left(s^{2}+p_{1} s\right) L[y]=\alpha s+\beta+\alpha p_{1}-p_{2} L[y] \\
& -p_{3} L[f(y)]+L[f(x)]
\end{aligned}
$$

or

$$
\begin{aligned}
L[y] & =\frac{\left(\alpha s+\beta+\alpha p_{1}\right)}{\left(s^{2}+p_{1} s\right)}-\frac{p_{2}}{\left(s^{2}+p_{1} s\right)} L[y] \\
& -\frac{p_{3}}{\left(s^{2}+p_{1} s\right)} L[f(y)]+L[f(x)]
\end{aligned}
$$

Taking inverse Laplace transform, we have

$$
\begin{aligned}
y(x) & =F(x)-L^{-1}\left(\frac{p_{2}}{s^{2}+p_{1} s} L[y]\right) \\
& -L^{-1}\left(\frac{p_{3}}{s^{2}+p_{1} s} L[f(y)]\right)
\end{aligned}
$$

where $F(x)$ represents the term arising from the source term and the prescribed initial conditions.

Now, we apply homotopy perturbation method [51],

$$
y(x)=\sum_{n=0}^{\infty} p^{n} y_{n}(x)
$$

where the term $y_{n}$ are to recursively calculated and the nonlinear term $f(y)$ can be decomposed as

$$
f(y)=\sum_{n=0}^{\infty} p^{n} H_{n}(y)
$$


for some He's polynomial $H_{n}$ (see [50,52]) that are given by

$$
\begin{aligned}
& H_{n}\left(y_{0}, y_{1}, y_{2}, \cdots, y_{n}\right) \\
& =\frac{1}{n !} \frac{\partial^{n}}{\partial p^{n}}\left[f\left(\sum_{i=0}^{\infty} p^{i} y_{i}\right)\right]_{p=0} \quad n=0,1,2,3 \cdots
\end{aligned}
$$

Substituting Equations (18) and (19) in (17), we get

$$
\begin{aligned}
& \sum_{n=0}^{\infty} p^{n} y_{n}(x)=F(x) \\
& -p\left(L^{-1}\left\{\frac{p_{2}}{\left(s^{2}+p_{1} s\right)} L\left[\sum_{n=0}^{\infty} p^{n} y_{n}(x)\right]\right\}\right. \\
& \left.+L^{-1}\left\{\frac{p_{3}}{\left(s^{2}+p_{1} s\right)} L\left[\sum_{n=0}^{\infty} p^{n} H_{n}(y)\right]\right\}\right)
\end{aligned}
$$

which is the coupling of the Laplace transformation and the homotopy perturbation method using He's polynomials. Comparing the coefficient of like powers of $p$, the following approximations are obtained:

$$
\begin{aligned}
p^{0}: \quad y_{0}(x)= & F(x), \\
p^{1}: \quad y_{1}(x)= & -\left(L^{-1}\left\{\frac{p_{2}}{\left(s^{2}+p_{1} s\right)} L\left[y_{0}(x)\right]\right\}\right. \\
& \left.+L^{-1}\left\{\frac{p_{3}}{\left(s^{2}+p_{1} s\right)} L\left[H_{0}(y)\right]\right\}\right) \\
p^{2}: y_{2}(x)=- & \left(L^{-1}\left\{\frac{p_{2}}{\left(s^{2}+p_{1} s\right)} L\left[y_{1}(x)\right]\right\}\right. \\
+ & \left.L^{-1}\left\{\frac{p_{3}}{\left(s^{2}+p_{1} s\right)} L\left[H_{1}(y)\right]\right\}\right) \\
y_{3}^{3}(x)=- & \left(L^{-1}\left\{\frac{p_{2}}{\left(s^{2}+p_{1} s\right)} L\left[y_{2}(x)\right]\right\}\right. \\
+ & \left.L^{-1}\left\{\frac{p_{3}}{\left(s^{2}+p_{1} s\right)} L\left[H_{2}(y)\right]\right\}\right)
\end{aligned}
$$

Example 2.1. Consider the following nonlinear PDE [53]:

$$
\nabla^{2} u+\left(\frac{\partial u}{\partial y}\right)^{2}=2 y+x^{4}
$$

with the following conditions:

$$
\begin{array}{lr}
u(x, 0)=a x, & u(x, 1)=x(x+a), \\
u(0, y)=0, & u(1, y)=y+a .
\end{array}
$$

Equation (22) can be written as

$$
\frac{\partial^{2} u}{\partial x^{2}}+\frac{\partial^{2} u}{\partial y^{2}}+\left(\frac{\partial u}{\partial y}\right)^{2}=2 y+x^{4}
$$

By applying the Laplace transform to both sides of Equation (24) subject to the initial condition, we have

$$
L[u]=\frac{1}{s^{2}}\left(L[2 y]+L\left[x^{4}\right]\right)-\frac{1}{s^{2}} L\left(u_{y y}+u_{y}^{2}\right)
$$

The inverse of the Laplace transform implies that

$$
\begin{aligned}
u(x, y) & =x^{2} y+\frac{x^{6}}{30} \\
& -L^{-1}\left(\frac{1}{s^{2}} L\left[u_{y y}\right]+\frac{1}{s^{2}} L\left[u_{y}^{2}\right]\right)
\end{aligned}
$$

Now, we apply the homotopy perturbation method, we have

$$
\begin{aligned}
& \sum_{n=0}^{\infty} p^{n} u_{n}(x, y)=x^{2} y+\frac{x^{6}}{30} \\
& -p L^{-1}\left(\frac{1}{s^{2}}\left\{L\left[\sum_{n=0}^{\infty} p^{n} u_{n}\right]+L\left[\sum p^{n} H_{n}(u)\right]\right\}\right)
\end{aligned}
$$

where $H_{n}(u)$ are He's polynomials. The first few components of He's polynomials are given by

$$
\begin{aligned}
& H_{0}(u)=y_{0 y}^{2}=x^{2} \\
& H_{1}(u)=2 y_{0 y} y_{1 y}=0 \\
& H_{2}(u)=y_{1 y}^{2}+2 y_{0 y} y_{2 y}=0
\end{aligned}
$$

Comparing the coefficient of like powers of $p$, we have

$$
\begin{aligned}
p^{0}: & u_{0}(x, y)=x^{2} y+\frac{x^{6}}{30}, \text { but we consider } \\
u_{0}(x, y) & =x^{2} y+\frac{x^{6}}{30}+a x \\
& p^{1}: u_{1}(x, y) \\
= & -L^{-1}\left[\frac{1}{s^{2}}\left\{L\left[y_{0 y y}\right]+L\left[H_{0}(y)\right]\right\}\right]=-\frac{x^{6}}{30} \\
& p^{2}: \quad u_{2}(x, y) \\
= & -L^{-1}\left[\frac{1}{s^{2}}\left\{L\left[y_{1 y y}\right]+L\left[H_{1}(y)\right]\right\}\right]=0 \\
& p^{3}: \quad u_{3}(x, y) \\
= & -L^{-1}\left[\frac{1}{s^{2}}\left\{L\left[y_{2 y y}\right]+L\left[H_{2}(y)\right]\right\}\right]=0
\end{aligned}
$$

So that the solution $u(x, y)$ is given by

$$
\begin{aligned}
u(x, y) & =u_{0}+u_{1}+u_{2}+u_{3}+\cdots \\
& =x^{2} y+\frac{x^{6}}{30}+a x-\frac{x^{6}}{30}+0+0+\cdots \\
& =x^{2} y+a x
\end{aligned}
$$

which is the exact solution of the problem.

Example 2.2. Consider the following non-homogeneous 
nonlinear PDE [53]:

$$
\frac{\partial u}{\partial t}=x^{2}-\frac{1}{4}\left(\frac{\partial u}{\partial x}\right)^{2}
$$

with the following condition:

$$
u(x, 0)=0
$$

By applying the Laplace transform method subject to the initial condition, we have

$$
y(x, s)=\frac{L\left[x^{2}\right]}{s}-\frac{1}{4 s} L\left[u_{x}^{2}\right]
$$

The inverse of the Laplace transform implies that

$$
u(x, t)=x^{2} t-L^{-1}\left[\frac{1}{4 s} L\left[u_{x}^{2}\right]\right]
$$
have

Now, we apply the homotopy perturbation method, we

$$
\begin{aligned}
& \sum_{n=0}^{\infty} p^{n} u_{n}(x, t) \\
& =x^{2} t-p\left(L^{-1}\left[\frac{1}{4 s}\left\{L\left[\sum_{n=0}^{\infty} p^{n} H_{n}(u)\right]\right\}\right]\right)
\end{aligned}
$$

where $H_{n}(u)$ are He's polynomials. The first few components of He's polynomials are given by

$$
\begin{aligned}
& H_{0}(u)=u_{0 x}^{2}=4 x^{2} t^{2} \\
& H_{1}(u)=2 u_{0 x} u_{1 x}=-\frac{8 x^{2} t^{4}}{3} \\
& H_{2}(u)=u_{1 x}^{2}+2 u_{0 x} u_{2 x}=\frac{4 x^{2} t^{6}}{9}+\frac{16 x^{2} t^{6}}{15}
\end{aligned}
$$
have

$$
\begin{array}{ll}
p^{0}: & u_{0}(x, t)=x^{2} t \\
p^{1}: & u_{1}(x, t)=-L^{-1}\left[\frac{1}{4 s}\left\{L\left[H_{0}(u)\right]\right\}\right]=-\frac{x^{2} t^{3}}{3} \\
p^{2}: & u_{2}(x, t)=-L^{-1}\left[\frac{1}{4 s}\left\{L\left[H_{1}(u)\right]\right\}\right]=\frac{2 x^{2} t^{5}}{15}
\end{array}
$$

Proceeding in a similar manner, we have

$$
p^{3}: \quad u_{3}(x, t)=-\frac{17 x^{2} t^{7}}{315}
$$

So that the solution $u(x, t)$ is given by

$$
\begin{aligned}
u(x, t) & =u_{0}+u_{1}+u_{2}+u_{3}+\cdots \\
& =x^{2} t-\frac{x^{2} t^{3}}{3}+\frac{2 x^{2} t^{5}}{15}-\frac{17 x^{2} t^{7}}{315}+\cdots
\end{aligned}
$$

\section{Variational Iteration Method (VIM)}

To illustrate the basic concept of the technique, we consider the following general differential equation

$$
L u+N u=g(x)
$$

where $L$ is a linear operator, $N$ is a nonlinear operator and $g(x)$ is the forcing term. According to VIM, we can construct a correct functional as follows

$$
u_{n+1}(x)=u_{n}(x)+\int_{0}^{x} \lambda\left(L u_{n}(s)+N \tilde{u}_{n}(s)-g(s)\right) \mathrm{d} s
$$

where $\lambda$ is a Lagrange multiplier. The subscripts $n$ denote the nth approximation, $\tilde{u}_{n}$ is considered as a restricted variation i.e. $\delta \tilde{u}_{n}=0$. In this method, it is required first to determine the Lagrange multiplier $\lambda$ optimally. The successive approximation $u_{n+1}, n \geq 0$ of the solution $u$ will be readily obtained upon using the determined Lagrange multiplier and any selective function $u_{0}$, consequently, the solution is given by $u=\lim _{n \rightarrow \infty} u_{n}$.

Now, we consider the following examples:

Example 3.1. Consider the following first order nonlinear differential equation [53]:

$$
\begin{aligned}
& y^{\prime}+y^{2}=0, \quad y \geq 0 \\
& y(0)=1
\end{aligned}
$$

If $y_{0}$ is an initial approximation or trial-function then we can write down following expression for correction:

$$
y_{n+1}(t)=y_{n}(t)+\int_{0}^{t} \lambda_{n}\left\{y_{n}^{\prime}(\tau)+y_{n}^{2}(\tau)\right\} \mathrm{d} \tau
$$

where the last term of right is called "correction", $\lambda_{n}$ is a general Lagrange multiplier. The above functional is called correction functional, the Lagrange multiplier in the functional should be such chosen that its correction solution is superior to its initial approximation (trialfunction) and is the best within the flexibility of the trialfunction, accordingly we can identified the multiplier by variational theory $[54,55]$. Making the above correction functional stationary with $y(0)=1$ so that, we can obtain following stationary conditions:

$$
\begin{aligned}
& -\lambda_{n}^{\prime}(\tau)+2 y_{n}(\tau) \lambda(\tau)=0 \\
& 1+\left.\lambda_{n}(\tau)\right|_{\tau=t}=0
\end{aligned}
$$

The Lagrange multiplier, therefore, can be identified as follows:

$$
\lambda_{n}(\tau)=-\exp \left(2 \int_{t}^{\tau} y_{n}(\xi) \mathrm{d} \xi\right)
$$

To simplify the multiplier, we approximate Equation (44) as follows: 


$$
\lambda_{n}(\tau)=-\exp \left(2 \int_{t}^{\tau} y_{0}(\xi) \mathrm{d} \xi\right)
$$

Substituting Equation (45) in Equation (41) yields following variational iteration formula

$$
\begin{aligned}
& y_{n+1}(t)=y_{n}(t) \\
& -\int_{0}^{t} \exp \left(2 \int_{t}^{\tau} y_{0}(\xi) \mathrm{d} \xi\left\{y_{n}^{\prime}(\tau)+y_{n}^{2}(\tau)\right\}\right) \mathrm{d} \tau
\end{aligned}
$$

We start with by above iteration formula, we can obtain following results,

$$
\begin{aligned}
y_{1}(t) & =1-\int_{0}^{t} e^{2(\tau-t)} \mathrm{d} \tau \\
& =1-\frac{1}{2}\left(1-e^{-2 t}\right)=\frac{1}{2}\left(1+e^{-2 t}\right)
\end{aligned}
$$

$$
\begin{aligned}
& y_{2}(t) \\
& =\frac{1}{2}\left(1+e^{-2 t}\right)-\int_{0}^{t} e^{2(\tau-t)}\left\{-e^{-2 \tau}+\frac{1}{4}\left(1+e^{-2 \tau}\right)^{2}\right\} \mathrm{d} \tau \\
& =\frac{1}{2}\left(1+e^{-2 t}\right)-\int_{0}^{t} e^{2(\tau-t)}\left\{\frac{1}{4}-\frac{1}{2} e^{-2 \tau}+\frac{1}{4} e^{-4 \tau}\right\} \mathrm{d} \tau \\
& =\frac{1}{2}\left(1+e^{-2 t}\right)-\frac{1}{8}\left(1-e^{-2 t}\right) \\
& +\frac{1}{2} t e^{-2 t}-\frac{1}{8}\left(e^{-2 t}-e-4 t\right) \\
& =\frac{1}{2}\left(1+e^{-2 t}\right)+\frac{1}{2} t e^{-2 t}-\frac{1}{8}\left(1-e^{-4 t}\right)
\end{aligned}
$$

if, suppose, $y_{2}(t)$ is sufficient, the approximation at $x$ $=0.4$ is $y_{2}(0.4)=0.6678$, while its exact one is $y(0.4)=$ 0.6667 , the $0.17 \%$ accuracy is remarkably good in view of the crudeness of its initial approximation. The process can, in principle, be continued as far as desired, however, the resulting integrals quickly become very cumbersome, so some simplification in the process of identification of Lagrange multiplier will be discussed at below:

We re-consider the correction functional Equation (41) as follows:

$$
y_{n+1}(t)=y_{n}(t)+\int_{0}^{t} \lambda\left\{y_{n}^{\prime}(\tau)+y_{n}^{2}(\tau)\right\} d \tau
$$

Where the nonlinear term $y_{n}^{2}$ is considered as nonvariational variation or constrained variation [54], i.e. $\delta y_{n}^{2}=0$. The Lagrange multiplier, therefore, can be readily identified and the following variation iteration formula can be obtained:

$$
y_{n+1}(t)=y_{n}(t)-\int_{0}^{t}\left\{y_{n}^{\prime}(\tau)+y_{n}^{2}(\tau)\right\} \mathrm{d} \tau
$$

Putting $\mathrm{n}=0$, 1, in Equation (50), we can obtain following results.

$$
\begin{aligned}
& y_{1}(t)=1-\int_{0}^{t}(0+1) \mathrm{d} \tau=1-t \\
& y_{2}(t)=1-t-\int_{0}^{t}\left\{-1+(1-\tau)^{2}\right\} \mathrm{d} \tau=1-t+t^{2}-\frac{1}{3} t^{3}
\end{aligned}
$$

Similarly putting $n=2,3, \cdots, n-1$, the $n$th approximation can be obtained, which converges to its exact solution, a little more slowly due to the approximate identification of the Lagrange multiplier.

\section{Remark}

The variational iteration technique mentioned above can be readily extended to partial differential equations (PDEs). Here the author will illustrate its process.

Example 3.2. Consider the following equation [53]:

$$
\begin{array}{lr}
\nabla^{2} u+\left(\frac{\partial u}{\partial y}\right)^{2}=2 y+x^{4} \\
u(0, y)=0, & u(1, y)=y+a \\
u(x, 0)=a x, & u(x, 1)=x^{2}+a x
\end{array}
$$

which has the exact solution $u=x(x y+a)$.

According to Adomian [56], an approximate solution can be obtained [57].

$$
\begin{aligned}
& u=x(x y+a) \\
& +\frac{1}{2}\left\{y(y-1)\left(\frac{x^{4}}{2}+\frac{y+1}{3}\right)+\frac{x}{30}\left(x^{5}-1\right)\right\}
\end{aligned}
$$

It is obvious that the approximation does not satisfy its boundary conditions. In 1995, Liu [57] proposes a modified Adomian's method called weighted residual decomposition method, with such method, he obtained following approximation:

$$
u=x(x y+a)+\frac{1}{4}\left\{x y(y-1)\left(1-x^{3}\right)\right\}
$$

which satisfies all its boundary conditions and has more higher accuracy than Adomian's. In 1978, Inokuti et al. [55] proposed a general Lagrange multiplier method to solve nonlinear mathematical physics which was first applied to quantum mechanics. In this method, a more accurate solution, depending upon its trial-function can be obtained for some special points, but not an approximate analytical one. J. H. He [53] tries to solve it by variational iteration method as follows:

Supposing the initial approximation of Equation (51) is $u_{0}$, its correction variational functional in $x$-direction and $y$-direction can be expressed respectively as follows:

$$
\begin{aligned}
& u_{n+1}(x, y)=u_{n}(x, y)+\int_{0}^{x} \lambda_{1}\left[\frac{\partial^{2} u_{n}(\xi, y)}{\partial \xi^{2}}\right. \\
& \left.+\frac{\partial^{2} \hat{u}_{n}(\xi, y)}{\partial y^{2}}+\left(\frac{\partial \hat{u}_{n}(\xi, y)}{\partial y}\right)^{2}-2 y-\xi^{4}\right] \mathrm{d} \xi
\end{aligned}
$$




$$
\begin{aligned}
& u_{n+1}(x, y)=u_{n}(x, y)+\int_{0}^{y} \lambda_{2}\left[\frac{\partial^{2} u_{n}(x, \varsigma)}{\partial \varsigma^{2}}\right. \\
& \left.+\frac{\partial^{2} \hat{u}_{n}(x, \varsigma)}{\partial x^{2}}+\left(\frac{\partial \hat{u}_{n}(x, \varsigma)}{\partial \varsigma}\right)^{2}-2 \varsigma-x^{4}\right] \mathrm{d} \varsigma
\end{aligned}
$$

where $\hat{u}_{n}$ is a nonvariational variation. Their stationary conditions are written down respectively as follows

$$
\frac{\partial^{2} \lambda_{1}(\xi)}{\partial \xi^{2}}=0,\left.\quad \lambda_{1}(\xi)\right|_{\xi=x}=0, \quad 1-\left.\frac{\partial \lambda_{1}(\xi)}{\partial \xi}\right|_{\xi=x}=0
$$

and

$$
\frac{\partial^{2} \lambda_{2}(\varsigma)}{\partial \varsigma^{2}}=0,\left.\quad \lambda_{2}(\varsigma)\right|_{\varsigma=y}=0, \quad 1-\left.\frac{\partial \lambda_{2}(\varsigma)}{\partial \varsigma}\right|_{\varsigma=y}=0
$$

The Lagrange multipliers can be easily identified:

$$
\lambda_{1}=\xi-x, \quad \lambda_{2}=\varsigma-y
$$

The iteration formulae in $x$-direction and $y$-directions can be, therefore, expressed respectively as follows

$$
\begin{aligned}
& u_{n+1}(x, y)=u_{n}(x, y)+\int_{0}^{x}(\xi-x)\left[\frac{\partial^{2} u_{n}(\xi, y)}{\partial \xi^{2}}\right. \\
& \left.+\frac{\partial^{2} u_{n}(\xi, y)}{\partial y^{2}}+\left(\frac{\partial u_{n}(\xi, y)}{\partial y}\right)^{2}-2 y-\xi^{4}\right] \mathrm{d} \xi \\
& u_{n+1}(x, y)=u_{n}(x, y)+\int_{0}^{y}(\varsigma-y)\left[\frac{\partial^{2} u_{n}(x, \varsigma)}{\partial \varsigma^{2}}\right. \\
& \left.+\frac{\partial^{2} u_{n}(x, \varsigma)}{\partial x^{2}}+\left(\frac{\partial u_{n}(x, \varsigma)}{\partial \varsigma}\right)^{2}-2 \varsigma-x^{4}\right] \mathrm{d} \varsigma
\end{aligned}
$$

To ensure the approximations satisfy the boundary conditions at $x=1$ and $y=1$, we modify the variational iteration formulae in $x$-direction and $y$-direction as follows:

$$
\begin{aligned}
& u_{n+1}(x, y)=u_{n}(x, y)+\int_{1}^{x}(\xi-x)\left[\frac{\partial^{2} u_{n}(\xi, y)}{\partial \xi^{2}}\right. \\
& \left.+\frac{\partial^{2} u_{n}(\xi, y)}{\partial y^{2}}+\left(\frac{\partial u_{n}(\xi, y)}{\partial y}\right)^{2}-2 y-\xi^{4}\right] \mathrm{d} \xi \\
& u_{n+1}(x, y)=u_{n}(x, y)+\int_{1}^{y}(\varsigma-y)\left[\frac{\partial^{2} u_{n}(x, \varsigma)}{\partial \varsigma^{2}}\right. \\
& \left.+\frac{\partial^{2} u_{n}(x, \varsigma)}{\partial x^{2}}+\left(\frac{\partial u_{n}(x, \varsigma)}{\partial \varsigma}\right)^{2}-2 \varsigma-x^{4}\right] \mathrm{d} \varsigma
\end{aligned}
$$

Now we start with an arbitrary initial approximation: $u_{0}=A+B x$, where $A$ and $B$ are constants to be determined, by the variational iteration formula in $x$-direction
(59), we have

$$
\begin{aligned}
u_{1}(x, y) & =A+B x+\int_{0}^{x}(\xi-x)\left[0+0-2 y-\xi^{4}\right] \mathrm{d} \xi \\
& =A+B x+x^{2} y+\frac{1}{30} x
\end{aligned}
$$

By imposing the boundary conditions at $x=0$ and $x=$ 1 yields $A=0$ and $B=a-1 / 30$, thus we have

$$
u_{1}(x, y)=x(x y+a)+\frac{1}{30} x\left(x^{5}-1\right)
$$

By (61), we have

$$
\begin{aligned}
u_{2}(x, y) & =x(x y+a)+\frac{1}{30} x\left(x^{5}-1\right) \\
+\int_{1}^{x}(\xi-x) & {\left[2 y+\xi^{4}+0+\xi^{4}-2 y-\xi^{4}\right] \mathrm{d} \xi } \\
= & x(x y+a)
\end{aligned}
$$

which is an exact solution. The approximation can also be obtained by $y$-direction. [53]:

Example 3.3. Consider the following nonlinear PDE

$$
\begin{gathered}
\frac{\partial u}{\partial t}=x^{2}-\frac{1}{4}\left(\frac{\partial u}{\partial x}\right)^{2} \\
u(x, 0)=0
\end{gathered}
$$

Its t-direction correction functional can be constructed as

$$
\begin{aligned}
& u_{n+1}(x, t)=u_{n}(x, t) \\
& +\int_{0}^{t} \lambda\left[\frac{\partial u_{n}(x, \tau)}{\partial \tau}-x^{2}+\frac{1}{4}\left(\frac{\partial \hat{u}_{n}(x, \tau)}{\partial x}\right)^{2}\right] \mathrm{d} \tau
\end{aligned}
$$

In which $\hat{u}_{n}$ is nonvariational variation. The multiplier can be identified and its variational iteration formula $t$-direction can be obtained

$$
\begin{aligned}
& u_{n+1}(x, t)=u_{n}(x, t) \\
& -\int_{0}^{t}\left[\frac{\partial u_{n}(x, \tau)}{\partial \tau}-x^{2}+\frac{1}{4}\left(\frac{\partial u_{n}(x, \tau)}{\partial x}\right)^{2}\right] \mathrm{d} \tau
\end{aligned}
$$

We start with initial approximation $u_{0}=0$, by above iteration formula, we can obtain successively its approximation:

$$
\begin{aligned}
u_{1}(x, t) & =0-\int_{0}^{t}\left(-x^{2}\right) \mathrm{d} \tau=x^{2} t \\
u_{2}(x, t) & =x^{2} t-\int_{0}^{t}\left[x^{2}-x^{2}+\frac{1}{4}(2 x \tau)^{2}\right] \mathrm{d} \tau \\
& =x^{2} t-\frac{1}{3} x^{2} t^{3}
\end{aligned}
$$




$$
\begin{aligned}
& u_{3}(x, t) \\
& =x^{2} t-\frac{1}{3} x^{2} t^{3}-\int_{0}^{t}\left[x^{2}-x^{2} t^{2}-x^{2}+\frac{1}{4}\left(2 x \tau-\frac{2}{3} x \tau^{3}\right)^{2}\right] \mathrm{d} \tau \\
& =x^{2} t-\frac{1}{3} x^{2} t^{3}-\int_{0}^{t}\left[-\frac{2}{3} x^{2} \tau^{4}-\frac{1}{9} x^{2} \tau^{6}\right] \mathrm{d} \tau \\
& =x^{2} t-\frac{1}{3} x^{2} t^{3}+\frac{2}{15} x^{2} t^{3}-\frac{1}{63} x^{2} t^{7}
\end{aligned}
$$

which is the same as Adomian's [56,58].

\section{Comparision of Variational Iterational Method and He-Laplace Method}

Example 4.1. Consider the following first order nonlinear differential equation [53]:

$$
y^{\prime}+y^{2}=0, y \geq 0
$$

with the following condition:

$$
y(0)=1 \text {. }
$$

By applying the aforesaid method subject to the initial condition, we have

$$
L[y(x)]=\frac{1}{s}-\frac{1}{s} L\left[y^{2}\right]
$$

The inverse of the Laplace transform implies that

$$
y(x)=1-L^{-1}\left[\frac{1}{s} L\left[y^{2}\right]\right]
$$

Now, we apply the homotopy perturbation method, we have

$$
\sum_{n=0}^{\infty} p^{n} y_{n}(x)=1-p\left(L^{-1}\left[\frac{1}{s} L\left[\sum_{n=0}^{\infty} p^{n} H_{n}(y)\right]\right]\right)
$$

where $H_{n}(y)$ are He's polynomials. The first few components of He's polynomials are given by

$$
\begin{aligned}
& H_{0}(y)=y_{0}^{2}=1 \\
& H_{1}(y)=2 y_{0} y_{1}=-2 x \\
& H_{2}(y)=y_{1}^{2}+2 y_{0} y_{2}=3 x^{2}
\end{aligned}
$$

Comparing the coefficient of like powers of $p$, we have

$$
\begin{array}{ll}
p^{0}: & y_{0}(x)=1 \\
p^{1}: & y_{1}(x)=-L^{-1}\left[\frac{1}{s}\left\{L\left[H_{0}(y)\right]\right\}=-x\right. \\
p^{2}: & y_{2}(x)=-L^{-1}\left[\frac{1}{s}\left\{L\left[H_{1}(y)\right]\right\}\right]=x^{2} \\
p^{3}: & y_{3}(x)=-L^{-1}\left[\frac{1}{s}\left\{L\left[H_{2}(y)\right]\right\}\right]=-x^{3}
\end{array}
$$

Table 1. Numerical results of Example 4.1.

\begin{tabular}{cccccc}
\hline$x$ & $\begin{array}{c}y_{\text {appx.3 }}(x) \\
\text { He-Laplace } \\
\text { method }\end{array}$ & $\begin{array}{c}y_{\text {appx.3 }}(x) \\
\text { VIM }\end{array}$ & $\begin{array}{c}y(x) \\
\text { exact }\end{array}$ & $\begin{array}{c}\text { Relative } \\
\text { error of } \\
\text { He-Laplace } \\
\text { Method }\end{array}$ & $\begin{array}{c}\text { Relative } \\
\text { error of VIM }\end{array}$ \\
\hline 0.05 & 0.9523750 & 0.9524583 & 0.9523809 & $6.1950 \mathrm{E}-06$ & $8.1270 \mathrm{E}-05$ \\
0.1 & 0.9090000 & 0.9096667 & 0.9090909 & $9.9990 \mathrm{E}-05$ & $6.3338 \mathrm{E}-04$ \\
0.15 & 0.8691250 & 0.8713750 & 0.8695652 & $5.0623 \mathrm{E}-04$ & $2.0812 \mathrm{E}-03$ \\
0.2 & 0.8320000 & 0.8373333 & 0.8333333 & $1.5999 \mathrm{E}-03$ & $4.8000 \mathrm{E}-03$ \\
0.25 & 0.7968750 & 0.8072917 & 0.8000000 & $3.9062 \mathrm{E}-03$ & $9.1146 \mathrm{E}-03$ \\
0.3 & 0.7630000 & 0.7810000 & 0.7692308 & $8.1000 \mathrm{E}-03$ & $1.5299 \mathrm{E}-02$ \\
0.35 & 0.7296250 & 0.7582083 & 0.7407407 & $1.5006 \mathrm{E}-02$ & $2.3581 \mathrm{E}-02$ \\
0.4 & 0.6960000 & 0.7386667 & 0.7142857 & $2.5599 \mathrm{E}-02$ & $3.4133 \mathrm{E}-02$ \\
\hline
\end{tabular}

So that the solution $y(x)$ is given by

$$
\begin{gathered}
y(x)=y_{0}+y_{1}+y_{2}+y_{3}+\cdots \\
y_{\text {appx. } 3}=1-x+x^{2}-x^{3}+\cdots
\end{gathered}
$$

which is converging to $\left(\frac{1}{(1+x)}\right)$ i.e. exact solution.

The computational results are presented in Table $\mathbf{1 .}$

\section{Conclusion}

In this paper, variational iteration method is employed for solving nonlinear ordinary and partial differential equations. The same problems are solved by He-Laplace method. It is worth mentioning that the He-Laplace method is capable of reducing the volume of the computational work as compared to the variational iteration methods while still maintaining the high accuracy of the numerical results.

\section{REFERENCES}

[1] A. M. Lyapunov, "The General Problem of the Stability of Motion," Taylor \& Francis, London, 1992

[2] J. Saberi-Nadjafi and A. Ghorbani, "He's Homotopy Perturbation Method: An Effective Tool for Solving Nonlinear Integral and Integro-Differential Equations," Computers and Mathematics with Applications, Vol. 58, No. 11-12, 2009, pp. 1345-1351. doi:10.1016/j.camwa.2009.03.032

[3] N. H. Sweilam and M. M. Khadar, "Exact Solutions of Some Coupled Nonlinear Partial Differential Equations Using the Homotopy Perturbation Method," Computers and Mathematics with Applications, Vol. 58, No. 11-12, 2009, pp. 2134-2141. doi:10.1016/j.camwa.2009.03.059

[4] A. V. Karmishin, A. I. Zhukov and V. G. Kolosov, "Methods of Dynamic Calculation and Testing for ThinWalled Structure," Mashinostroyenie, Moscow, 1990.

[5] R. Hirota, "Exact Solutions of the Korteweg-deVries Equation for Multiple Collisions of Solitons," Physics Review Letters, Vol. 27, No. 18, 1971, pp. 1192-1194. 
doi:10.1103/PhysRevLett.27.1192

[6] A. M. Wazwaz, "On Multiple Soliton Solution for Coupled KdV-mkdV Equation," Nonlinear Science Letter A, Vol. 1, 2010, pp. 289-296.

[7] G. Adomian, "Solving Frontier Problems of Physics: The Decomposition Method," Kluwer Academic Publication, Boston, 1994.

[8] G. C. Wu and J. H. He, "Fractional Calculus of Variations in Fractal Space Time," Nonlinear Science Letter A, Vol. 1, 2010, pp. 281-287. doi:10.1515/IJNSNS.2010.11.S1.281

[9] M. A. Abdou and A. A. Soliman, "New Application of Variational Iteration Method," Physica D: Nonlinear Phenomena, Vol. 211, No. 1-2, 2005, pp. 1-8. doi:10.1016/j.physd.2005.08.002

[10] C. Chun, "Fourier-Series Based Variational Iterationmethod for a Reliable Treatment of Heat Equations with Variable Coefficients," International Journal of Nonlinear Sciences and Numerical Simulation, Vol. 10, 2009, pp. 1383-1388. doi:10.1515/IJNSNS.2009.10.11-12.1383

[11] N. Faraz, Y. Khan and A. Yildirim, "Analytical Approach to Two-Dimensional Viscous Flow with a Shrinking Sheet via Variational Iteration Algorithm-II," Journal of King Saud University, Vol. 23, No. 1, 2010, pp. 1-120. doi:10.1016/j.jksus.2010.06.010.

[12] J. H. He, "Variational Iteration Method-A Kind of Nonlinear Analytical Technique: Some Examples," International Journal of Nonlinear Mechanics, Vol. 34, No. 4, 1999, pp. 699-708. doi:10.1016/S0020-7462(98)00048-1

[13] J. H. He and X. H. Wu, "Variational Iteration Method: New Development and Applications," Computers and Mathematics with Applications, Vol. 54, 2007, pp. 881894. doi:10.1016/j.camwa.2006.12.083

[14] J. H. He, G. C. Wu and F. Austin, "The Variational Iteration Method Which Should Be Followed," Nonlinear Science Letter A, Vol. 1, No. 1, 2009, pp. 1-30.

[15] E. Hesameddini and H. Latifizadeh, "Reconstruction of Variational Iteration Algorithm Using the Laplace Transform," International Journal of Nonlinear Sciences and Numerical Simulation, Vol. 10, 2009, pp. 1377-1382. doi:10.1515/IJNSNS.2009.10.11-12.1377

[16] L. A. Soltani and A. Shirzadi, "A New Modification of the Variational Iteration Method," Computers and Mathematics with Applications, Vol. 59, No. 1, 2010, pp. 25282535. doi:10.1016/j.camwa.2010.01.012

[17] G. C. Wu and E. W. M. Lee, "Fractional Variational Iteration Method, and Its Application," Physics Letter A, Vol. 374, No. 25, 2010, pp. 2506-2509. doi:10.1016/j.physleta.2010.04.034

[18] G. Adomian, "Solution of Physical Problems by Decomposition," Computers and Mathematics with Applications, Vol. 2, No. 9-10, 1994, pp. 145-154. doi:10.1016/0898-1221(94)90132-5

[19] D. Bahuguna, A. Ujlayan and D. N. Pandey, "Acomparative Study of Numerical Methods for Solving an IntegroDifferential Equation," Computers and Mathematics with Applications, Vol. 57, 2009, pp. 1485-1493. doi:10.1016/j.camwa.2008.10.097

[20] M. Dehghan, "Weighted Finite Difference Techniques for the One Dimensional Advection-Diffusion Equation," Applied Mathematics and Computation, Vol. 147, No. 2, 2004, pp. 307-319. doi:10.1016/S0096-3003(02)00667-7

[21] D. D. Ganji and A. Sadighi, “Application of He's Homotopy Perturbation Method to Nonlinear Coupled Systems of Reaction-Diffusion Equations," International Journal of Nonlinear Sciences and Numerical Simulation, Vol. 7, 2006, pp. 411-418. doi:10.1515/IJNSNS.2006.7.4.411

[22] D. D. Ganji, “The Application of He's Homotopy Perturbation Method to Nonlinear Equation Arising in Heat Transfer," Physics Letter A, Vol. 335, No. 2, 2006, pp. 337-341. doi:10.1016/i.physleta.2006.02.056

[23] H. K. Mishra and A. K. Nagar, "He-Laplace Method for Linear and Nonlinear Partial Differential Equations," Journal of Applied Mathematics, Vol. 2012, 2012, pp. 1-16. doi:10.1155/2012/180315

[24] S. T. Mohyud-Din, M. A. Noor and K. I. Noor, "Travelling Wave Solutions of Seventh-Order Generalized KdV Equation Using He's Polynomials," International Journal of Nonlinear Sciences and Numerical Simulation, Vol. 10, 2009, pp. 227-233. doi:10.1515/IJNSNS.2009.10.2.227

[25] A. M. Wazwaz, "A Comparison between the Variational Iteration Method and Adomian Decomposetion Method," Journal of Computational and Applied Mathematics, Vol. 207, No. 1, 2007, pp. 129-136. doi:10.1016/j.cam.2006.07.018

[26] E. Yusufoglu, "Numerical Solution of Duffing Equation by the Laplace Decomposition Algorithm," Applied Mathematics and Computation, Vol. 177, No. 1, 2006, pp. 572-580.

[27] J. H. He, "Homotopy Perturbation Method: A New Nonlinear Analytical Technique," Applied Mathematics and Computation, Vol. 135, No. 1, 2003, pp. 73-79. doi:10.1016/S0096-3003(01)00312-5

[28] J. H. He, "Homotopy, Comparion of Homotopy Perturbation Method and Homotopy Analysis Method," Applied Mathematics and Computation, Vol. 156, 2004, pp. 527 539. doi:10.1016/j.amc.2003.08.008

[29] J. H. He, "Homotopy, the Homotopy Perturbation Method for Nonlinear Oscillators with Discontinueties," Applied Mathematics and Computation, Vol. 151, 2004, pp. 287292. doi:10.1016/S0096-3003(03)00341-2

[30] J. H. He, "Recent Developments of the Homotopy Perturbation Method," Topological Methods in Nonlinear Analysis, Vol. 31, 2008, pp. 205-209.

[31] J. H. He, "New Interpretation of Homotopy Perturbation Method," International Journal of Modern Physics, Vol. 20, No. 18, 2006, pp. 2561-2568. doi:10.1142/S0217979206034819

[32] J. H. He, "A Coupling Method of Homotopy Technique and a Perturbation Technique for Nonlinear Problems," International Journal of Nonlinear Mechanics, Vol. 35, 2000, pp. 37-43. doi:10.1016/S0020-7462(98)00085-7

[33] J. H. He, "Variational Iteration Method for Autonomous Ordinary Differential Systems," Applied Mathematics and Computation, Vol. 114, 2000, pp. 115-123. 


\section{doi:10.1016/S0096-3003(99)00104-6}

[34] J. H. He, "Homotopy Perturbation Technique," Computer methods in Applied Mechanics and Engineering, Vol. 178, No. 3-4, 1999, pp. 257-262. doi:10.1016/S0045-7825(99)00018-3

[35] J. H. He, "A Simple Perturbation Approach to Blasius Equation," Applied Mathematics and Computation, Vol. 140, No. 2-3, 2003, pp. 217-222. doi:10.1016/S0096-3003(02)00189-3

[36] J. H. He, "Application of Homotopy Perturbation Method to Nonlinear Wave Equation," Chaos, Solitons, Fractals, Vol. 26, No. 3, 2005, pp. 295-300. doi:10.1016/j.chaos.2005.03.006

[37] J. H. He, "Homotopy Perturbation Method for Solving Boundary Value Problem," Physics Letter A, Vol. 350, No. $1-2,2006$, pp. $87-88$. doi:10.1016/j.physleta.2005.10.005

[38] E. Hesameddini and H. Latifizadeh, "An Optimal Choice of Initial Solutions in the Homotopy Perturbation Method," International Journal of Nonlinear Sciences and Numerical Simulation, Vol. 10, 2009, pp. 1389-1398. doi:10.1515/IJNSNS.2009.10.11-12.1389

[39] E. Hesameddini and H. Latifizadeh, "A New Vision of the He's Homotopy Perturbation Method," International Journal of Nonlinear Sciences and Numerical Simulation, Vol. 10, 2009, pp. 1415-1424. doi:10.1515/IJNSNS.2009.10.11-12.1415

[40] M. Rafei and D. D. Ganji, "Explicit Solutions of Helmhotz Equation and Fifth-Order KdV Equation Using Homotopy Perturbation Method," International Journal of Nonlinear Sciences and Numerical Simulation, Vol. 7, 2006, pp. 321-328. doi:10.1515/IJNSNS.2006.7.3.321

[41] A. M. Siddiqui, R. Mohmood and Q. K. Ghori, "Thin Film Flow of a Third Grade Fluid on a Moving Belt by He's Homotopy Perturbation Method," International Journal of Nonlinear Sciences and Numerical Simulation, Vol. 7, No. 1, 2006, pp. 7-14. doi:10.1515/IJNSNS.2006.7.1.7

[42] L. Xu, "He's Homotopy Perturbation Method for a Boundary Layer Equation in Unbounded Domain," Computers and Mathematics with Applications, Vol. 54, No. 7-8, 2007, pp. 1067-1070. doi:10.1016/j.camwa.2006.12.052

[43] J. Biazar, M. Gholamiporshokuhi and B. Ghanbari, "Extracting a General Iterative Method from an Adomian Decomposition Method and Comparing It to the Variational Iteration Method," Computers and Mathematics with Applications, Vol. 59, No. 2, 2010, pp. 622-628. doi:10.1016/i.camwa.2009.11.001

[44] S. Islam, Y. Khan, N. Faraz and F. Austin, "Numerical Solution of Logistic Differential Equations by Using the Laplace Decomposition Method," World Applied Sciences Journal, Vol. 8, 2010, pp. 1100-1105.
[45] Y. Khan and F. Austin, "Application of the Laplace Decomposition Method to Nonlinear Homogeneous and Non-Homogeneous Advection Equations," Zeitschrift fuer Naturforschung, Vol. 65, 2010, pp. 1-5.

[46] Y. Khan and Q. B. Wu, "Homotopy Perturbation Transform Method for Nonlinear Equations Using He's Polynomials," Computers and Mathematics with Applications, Vol. 61, No. 8, 2011, pp. 1963-1967. doi:10.1016/j.camwa.2010.08.022

[47] Y. Khan, "An Effective Modification of the Lap-Lace Decomposition Method for Nonlinear Equations," International Journal of Nonlinear Sciences and Numerical Simulation, Vol. 10, 2009, pp. 1373-1376. doi:10.1515/IJNSNS.2009.10.11-12.1373

[48] S. A. Khuri, "A Laplace Decomposition Algorithm Applied to a Class of Nonlinear Differential Equations," Journal of Applied Mathematics, Vol. 1, No. 4, 2001, pp. 141-155. doi:10.1155/S1110757X01000183

[49] M. Madani and M. Fathizadeh, "Homotopy Perturbation Algorithm Using Laplace Transformation," Nonlinear Science Letters A, Vol. 1, 2010, pp. 263-267.

[50] A. Ghorbani, "Beyond Adomian's Polynomials: He's Polynomials," Chaos, Solitons, Fractals, Vol. 39, 2009, pp. 1486-1492. doi:10.1016/j.chaos.2007.06.034

[51] J. H. He, "Homotopy Perturbation Technique," Computer Methods in Applied Mechanics and Engineering, Vol. 178, No. 3-4, 1999, pp. 257-262. doi:10.1016/S0045-7825(99)00018-3

[52] S. T. Mohyud-Din and A. Yildirim, "Homotopy PerturbBation Method for Advection Problems," Nonlinear Science Letter A, Vol. 1, No. 3, 2010, pp. 307-312.

[53] J. H. He, "A New Approach to Nonlinear Partial Differential Equations," Communications in Nonlinear Science and Numerical Simulation, Vol. 2, 1997, pp. 230-235. doi:10.1016/S1007-5704(97)90007-1

[54] B. A. Finlayson, "The Method of Weighted Residuals and Variational Principles," Academic Press, London, 1972.

[55] M. Inokuti, et al., "General Use of the Lagrange Multiplier in Nonlinear Mathematical Physics," In: S. NematNasser, Ed., Variational Method in the Mechanics of Solids Pergamon, 1978, pp. 156-162.

[56] G. Adomian, "Stochastic System," Academic Press, London, 1983

[57] G. L. Liu, "Weighted Residual Decomposition Method in Nonlinear Applied Mathematics," Proceedings of 6th Congress of Modern Mathematics and Mechanics, Suzhou, 1995, pp. 643-648.

[58] G. Adomian, "A Review of the Decomposition Method in Applied Mathematics," Journal of Mathematical Analysis and Application, Vol. 135, No. 2, 1988, pp. 501-544. 